\title{
Survivin depletion inhibits tumor growth and enhances chemosensitivity in hepatocellular carcinoma
}

\author{
YVONNE Y.Y. OR ${ }^{1}$, ARIEL K.M. CHOW ${ }^{1,2}$, LUI NG ${ }^{1}$, SHEUNG TAT FAN ${ }^{1,2}$, \\ THOMAS C.C. YAU ${ }^{1}$, RONNIE T.P. POON ${ }^{1,2}$ and ROBERTA W.C. PANG ${ }^{1,2}$ \\ ${ }^{1}$ Department of Surgery, and ${ }^{2}$ Centre for Cancer Research, Li Ka Shing Faculty of Medicine, \\ The University of Hong Kong, Hong Kong, SAR, P.R. China
}

Received October 10, 2013; Accepted March 14, 2014

DOI: $10.3892 / \mathrm{mmr} .2014 .2413$

\begin{abstract}
Survivin is a member of the inhibitor of apoptosis family, which has been suggested to be crucial in the control of cell division and inhibition of apoptosis. Expression of this protein has been observed in transformed cell lines and human tumor tissues, including those from colorectal cancer, but not in terminally differentiated adult tissues. Survivin mRNA expression has frequently been detected in hepatocellular carcinoma (HCC) and its protein expression has been demonstrated to be highly correlated with proliferation index rather than apoptotic index. The present study aimed to analyze the effect of survivin on the tumorigenicity and chemosensitivity of HCC via the establishment of an HCC cell line (PLC/PRF/5) with the stable knockdown of the survivin gene (PLC-k3). This cell line displayed significantly lower rates of survival and proliferation in assays of cell viability and proliferation, respectively, compared with those of the control cell line (PLC-v). In addition, PLC-k3 cells were more sensitive to cisplatin treatment, resulting in $\mathrm{S}$ phase arrest. These findings were further confirmed by an in vivo experiment. The data of the present study suggest that survivin is critical in promoting cell proliferation but not in inhibition of apoptosis, and enhances the chemosensitivity of HCC. Thus, the suppression of survivin expression in combination with cisplatin may contribute to the development of more effective treatments for HCC.
\end{abstract}

\section{Introduction}

Hepatocellular carcinoma (HCC) is the fifth most common type of cancer in males and the seventh most common type of cancer in females, with a total of 0.7 million new cases

Correspondence to: Dr Roberta W.C. Pang, The University of Hong Kong, 21 Sassoon Road, Hong Kong, SAR, P.R. China

E-mail: robertap@hku.hk

Key words: chemosensitivity, cisplatin, hepatocellular carcinoma, survivin, tumorigenesis worldwide in 2008. HCC is the second and the sixth most common cause of cancer-related mortalities in males and females, respectively, with $>0.5$ million mortalities worldwide in 2008 (1). Due to the low five-year survival rate following surgery and the frequent chemoresistance that is observed in patients with HCC, the development of an effective treatment is required.

Survivin, encoded by the gene BIRC5, is a member of the inhibitor of apoptosis protein family, and has been implicated in the control of cell division and the inhibition of apoptosis (2,3). It inhibits apoptosis through binding with caspase-9, and accelerates mitotic activity via association with microtubules of the mitotic spindle in the $\mathrm{G} 2 / \mathrm{M}$ phase $(4,5)$. Survivin has been demonstrated to be selectively expressed during embryonic development, but with low or no expression in terminally differentiated adult tissues $(6,7)$. However, re-expression of the protein has been displayed in transformed cell lines and various types of human tumor tissues $(6,8)$. In addition, survivin expression is correlated with poor prognosis in various types of cancer, such as lung adenocarcinoma (8) and colorectal cancer $(10,11)$. In patients with HCC, tumor tissues have been demonstrated to express survivin mRNA $(87.5 \%)$, whereas no expression was detected in normal liver tissues and tissues from non-tumor areas (12). Survivin expression has also been demonstrated to be highly correlated with proliferation index in HCC (12-15). Due to the possible roles of survivin in the suppression of apoptosis and promotion of proliferation in tumor tissues, survivin was widely recognized as a critical therapeutic target. It is therefore hypothesized that survivin depletion leads to cell cycle arrest, reduction of cell proliferation, induction of apoptosis and increased drug sensitivity. These factors may prove critical for the development of cancer therapies. Therefore, the current study aimed to demonstrate the effect of survivin depletion on cell viability and tumor growth in HCC in vitro and in vivo.

\section{Materials and methods}

Cell culture. The HCC cell line (PLC/PRF/5; ATCC, Manassas, VA, USA) was maintained in Dulbecco's modified Eagle's medium (Invitrogen, Carlsbad, CA, USA) supplemented with $10 \%$ fetal bovine serum at $37^{\circ} \mathrm{C}$ in a $95 \%$ humidified incubator containing $5 \% \mathrm{CO}_{2}$. 
Cell transfection with antisense survivin. The pEGFP vector containing the antisense version of the survivin full-length coding sequence (OriGene, Rockville, MD, USA) was confirmed by sequencing, using an ABI Prism 3100 Genetic analyzer (Applied Biosystems, Foster City, CA, USA). The plasmid was transfected into the PLC/PRF/5 cells using FuGene ${ }^{\circledR} 6$ Transfection reagent (Roche Diagnostics, Indianapolis, IN, USA). The PLC/PRF/5 cells stably transfected with the survivin antisense sequence or empty pEGFP-N1 vectors (Clontech Laboratories, Mountain View, CA, USA) were maintained by continuous G418 (Roche Diagnostics) drug selection. Expression of the survivin gene was confirmed with western blot analysis of the stably transfected cells. Clones with the successfully transfected antisense sequence (PLC-k3) and empty vectors alone (PLC-v) were then used for further in vitro and in vivo analysis.

Treatment with cisplatin. In order to determine the effect of survivin depletion on drug sensitivity, PLC-k3 and PLC-v cells were treated with $3.5 \mathrm{mg} / \mathrm{ml}$ cisplatin for 24 and $48 \mathrm{~h}$. A cell proliferation assay, Annexin $\mathrm{V}$ apoptotic assay, and cell cycle analysis were then performed as subsequently described.

Cell proliferation assay. The proliferation rates of the untreated and cisplatin-treated PLC-k3 and PLC-v cells were determined by a 3-(4,5-dimethylthiazol-2-yl)-2,5-diphenyltetrazolium bromide (MTT; Sigma, St. Louis, MO, USA) cell proliferation assay. Cells were plated at 6,000 cells/well in 96-well culture plates. The MTT assay was performed at 0, 24, 48, 72 and $96 \mathrm{~h}$ on the untreated cells and at 24 and $48 \mathrm{~h}$ after treatment on the cisplatin-treated cells. Viability was assessed with the addition of MTT solution $(1 \mathrm{mg} / \mathrm{ml})$ and an average absorbance of $570 \mathrm{~nm}$ was determined from triplicate samples.

In addition, a trypan blue exclusion assay was performed to quantify the viable cells in the untreated and cisplatin-treated PLC-k3 and PLC-V cell groups. Cells were seeded at $2 \times 10^{4}$ cells/well in 24-well plates. A trypan blue exclusion assay was performed at $0,24,48$ and $72 \mathrm{~h}$ on untreated cells and at 24 and $48 \mathrm{~h}$ after treatment on cisplatin-treated cells by staining the trypsinized cells with $0.4 \%$ trypan blue. The number of unstained cells (viable cells) from triplicate samples was recorded using a hemocytometer (Marienfeld, Lauda-Königshofen, Germany).

Annexin V apoptotic assay. The effect of survivin depletion on cellular apoptosis was determined by the Annexin V PE and 7AAD Apoptosis Detection kit (BD Biosciences, San Jose, CA, USA) according to the manufacturer's instructions. Briefly, the untreated or the cisplatin-treated PLC-k3 and PLC-v cells were harvested, washed with phosphate-buffered saline, and stained with the Annexin V/7AAD mixture in binding buffer from the kit for $15 \mathrm{~min}$ at room temperature in the dark. The percentages of apoptotic cells were then determined using a FACSCalibur flow cytometer (BD Biosciences).

Cell cycle analysis. The untreated and cisplatin-treated PLC-k3 and PLC-v cells were harvested and fixed in cold $70 \%$ ethanol at $-20{ }^{\circ} \mathrm{C}$ for $24 \mathrm{~h}$. Fixed cells were then washed and incubated in $0.2 \mathrm{mg} / \mathrm{ml}$ propidium iodide and $0.2 \mathrm{mg} / \mathrm{ml}$ RNase $\mathrm{A}$ at $37^{\circ} \mathrm{C}$ for $30 \mathrm{~min}$ in the dark. The labeled cells were subjected to the FACSCalibur flow cytometer for cell cycle distribution analysis. The percentage of cells in each phase was analyzed using ModFit LT software (Verity Software House, Inc., Topsham, ME, USA).

Immunoblotting. PLC-k3 and PLC-v cells were then lysed in ice-cold radio-immunoprecipitation assay buffer containing $150 \mathrm{mM} \mathrm{NaCl}, 1 \mathrm{mM}$ EDTA, $1 \%$ (v/v) NP-40, 0.25\% (w/v) sodium deoxycholate, $1 \mathrm{mM}$ phenylmethanesulfonyl fluoride, and $1 \mathrm{U}$ protease inhibitor cocktail (Roche Diagnostics, Penzberg, Germany) in $50 \mathrm{mM}$ Tris- $\mathrm{HCl}$ buffer, $\mathrm{pH}$ 7.4. Equal amounts of protein were loaded onto an SDS-polyacrylamide gel under reducing conditions for gel electrophoresis and then transferred to a polyvinylidine fluoride membrane (Amersham Biosciences, Piscataway, NJ, USA). Blots were probed with the following antibodies: Anti-survivin rabbit polyclonal antibody (Calbiochem, San Diego, CA, USA), Anti-cyclin A rabbit polyclonal antibody, cyclin B1 rabbit polyclonal antibody and cyclin D1 rabbit polyclonal antibody (Cell Signaling Technology, Danvers, MA, USA), and the expression of $\beta$-actin ( $\beta$-actin mouse monoclonal antibody; Sigma-Aldrich, St. Louis, MO, USA) was used as a loading control. After probing with horseradish peroxidase-conjugated secondary antibodies, membranes were developed with the Immobilon Western Chemiluminescent HRP Substrate system (Millipore, Billerica, MA, USA). The signals were then captured by the ChemiDoc XRS+ system (Bio-Rad, Hercules, CA, USA) and analyzed using Image Lab (Bio-Rad).

In vivo studies. Experiments involving animals were approved by the Committee on the Use of Live Animals for Teaching and Research, University of Hong Kong (Hong Kong, China; no. 1731-08). BALB/c-nu/nu (nude) mice (Charles River Laboratories, Wilmington, MA, USA) were maintained in laminar flow cabinets under pathogen-free conditions, and all efforts were made to reduce suffering. PLC-k3 and PLC-v cells were harvested from mid-log phase cultures, and $1.5 \times 10^{6}$ cells were injected into the mice subcutaneously in order to induce xenograft tumor formation. Two weeks post-injection, the mice were randomly divided into the following 4 groups with 6 mice in each: PLC-v-(control), PLC-v-(cisplatin), PLC-k3-(control) and PLC-k3-(cisplatin). Cisplatin or saline $(3 \mathrm{mg} / \mathrm{kg}$ ) was administered intraperitoneally twice a week for 28 days. Caliper measurement of tumor dimensions was performed twice a week to estimate tumor size, with the formula $0.5 \times 1 \times \mathrm{w}^{2}$ where 1 is the length and $\mathrm{w}$ is the width of the tumor. Mice were sacrificed with sodium pentobarbitone $(150 \mathrm{mg} / \mathrm{kg})$ overdose on day 42.

Statistical analysis. Data are presented as the mean \pm standard deviation of three independent experiments for the in vitro study, and from six mice for the in vivo study. Data were analyzed using one-way analysis of variance, and $\mathrm{P}<0.05$ was considered to indicate a statistically significant difference.

\section{Results}

Knockdown of the survivin gene in the PLC/PRF/5 cells. The pEGFP vector containing the antisense sequence of the 
A

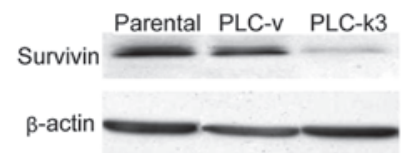

D

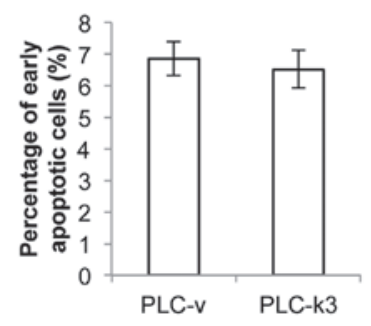

B

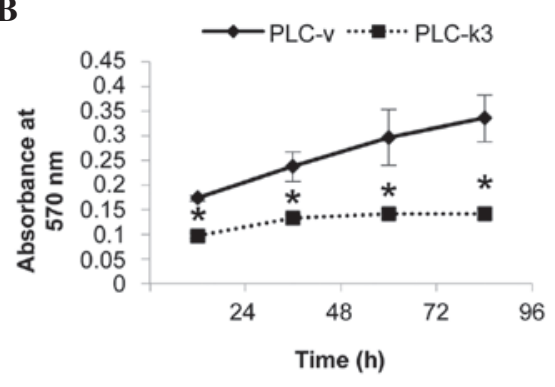

E

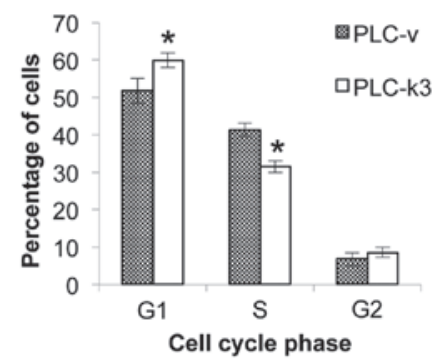

C

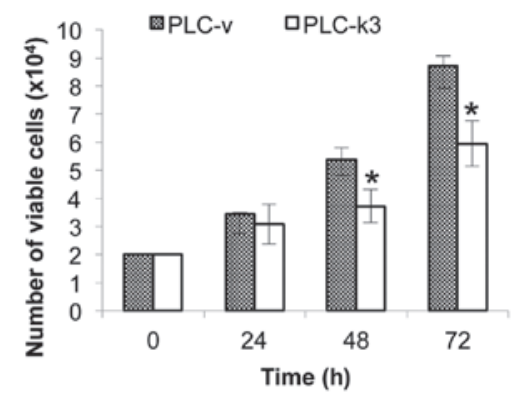

F

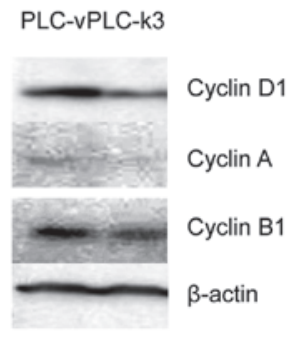

Figure 1. (A) Representative blot displaying the expression of survivin in different clones of PLC/PRF/5 cells. The viability of PLC-v and PLC-k3 cells was studied by (B) MTT assay and (C) trypan blue assay. Results are presented as the mean \pm standard deviation of three separate experiments. ${ }^{*} \mathrm{P}<0.05 \mathrm{PLC}-\mathrm{v}$ vs. PLC-k3 at the respective time point. Bar charts summarizing the percentages of PLC-v and PLC-k3 cells (D) in early apoptosis and (E) distributed in each phase of the cell cycle. Results are presented as the mean \pm standard deviation of three separate experiments. " $\mathrm{P}<0.05$ PLC-v vs. PLC-k 3 at the respective phase. (F) Representative blot displaying the expression of cyclin D1, A and B1 in PLC-v and PLC-k3 cells. $\beta$-actin was used as a loading control.

survivin gene was transfected into PLC/PRF/5 cells for functional experiments. Immunoblotting analysis of the PLC-v and PLC-k3 cells demonstrated the successful knockdown of survivin expression in the PLC-k3 cells (Fig. 1A).

Survivin depletion reduces viability of PLC/PRF/5 cells. The effect of survivin depletion on cell viability was examined with an MTT assay. In $96 \mathrm{~h}$, the average absorbance of the PLC-v cells increased by 2 -fold, while it increased by $40 \%$ in the PLC-k3 cells. The rate of the increase in the number of viable cells in the PLC-v group was significantly higher than that in PLC-k3 group (Fig. 1B).

In addition, a trypan blue assay was performed to quantify the viable cells in the PLC-v and PLC-k3 groups (Fig. 1C). The doubling time was 26.3 and $42.0 \mathrm{~h}$ for the PLC-v and PLC-k3 cells, respectively. The PLC-k3 cells demonstrated a significantly lower number of viable cells compared with that of the PLC-v cells at 48 (26\% lower) and $72 \mathrm{~h}$ ( $31 \%$ lower).

In order to determine whether cellular apoptosis or cell cycle progression contributed to the difference in the viability of the PLC-v and PLC-k3 cells, an Annexin V apoptotic assay and cell cycle analysis were performed. The results of the apoptotic assay indicated no significant difference in the percentages of early apoptotic PLC-v and PLC-k3 cells (Fig. 1D). The analysis of cell cycle progression revealed that the PLC-k3 group exhibited $8.12 \%$ more cells in the G1 phase and $9.89 \%$ fewer cells in the $\mathrm{S}$ phase, compared with those of the PLC-v group (Fig. 1E). This difference in cell cycle progression may contribute to the lower cell viability of the PLC-k3 cells.

The expression of cyclin D1, A and B1 was also investigated by immunoblotting. Reduced expression levels of cyclin D1, A and B1 were observed in the PLC-k3 cells compared with those in the PLC-v cells (Fig. 1F).
Survivin depletion enhances cisplatin sensitivity. The effect of survivin depletion on cisplatin sensitivity was also examined. The PLC-k3 and PLC-v cells were treated with cisplatin for 24 and $48 \mathrm{~h}$ and an MTT cell proliferation assay was performed (Fig. 2A). When cells were treated for $24 \mathrm{~h}$, cisplatin did not exert any effect on the viability of the PLC-v cells compared with that of the untreated cells, whereas a significant reduction of $45 \%$ was observed in the viability of the cisplatin-treated PLC-k3 cells compared with that of the untreated PLC-k3 cells. Following the 48-h treatment, a significant reduction was observed in the viability of the cisplatin-treated PLC-v cells ( $21 \%$ vs. the untreated PLC-v cells), and the cisplatin-treated PLC-k3 cells ( $47 \%$ vs. the untreated PLC-k3 cells).

Similarly, a trypan blue assay was performed to quantify the viable PLC-v and PLC-k3 cells following cisplatin treatment (Fig. 2B). Following the 24-h treatment, a significant reduction in the number of viable cells was observed in the cisplatin-treated PLC-v group (27\% vs. the untreated PLC-v cells) and the cisplatin-treated PLC-k3 group (33\% vs. the untreated PLC-k3 cells). Following the 48-h treatment, a significant reduction in the number of viable cells was observed in the cisplatin-treated PLC-v group ( $42 \%$ vs. the untreated PLC-v cells) and the cisplatin-treated PLC-k3 group (67\% vs. the untreated PLC-k3 cells). Therefore, the results of the trypan blue assay were in agreement with those of the MTT assay in demonstrating that the PLC-k3 cells were more sensitive to cisplatin treatment than the PLC-v cells.

In order to determine whether cellular apoptosis contributed to the differences in the viability of the PLC-v and PLC-k3 cells with cisplatin treatment, an Annexin V apoptotic assay was performed (Fig. 2C). Increases in the percentages of early apoptotic cells were observed in the PLC-v and PLC-k3 groups when treated with cisplatin. Following the 24-h treatment, a significant increase in the number of early apoptotic cells 
A
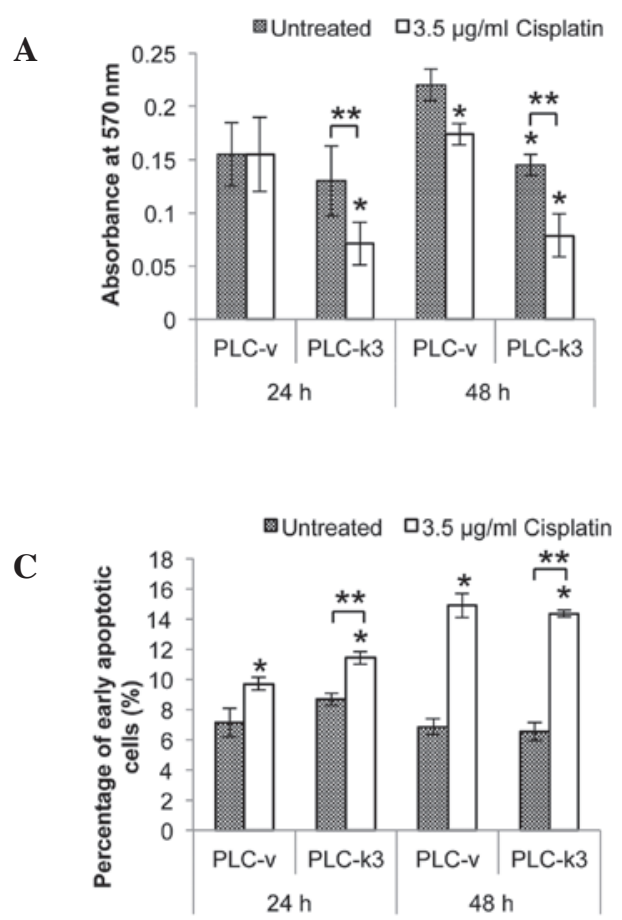

B

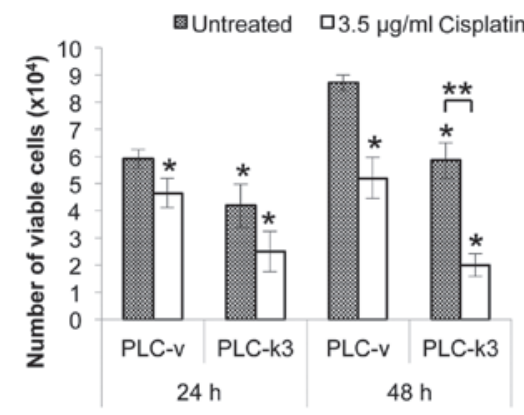

D

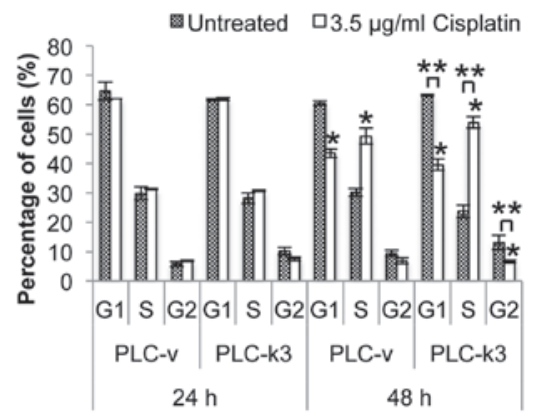

Figure 2. Following treatment with cisplatin, the viability, early apoptosis and cell cycle distribution of PLC-v and PLC-k3 cells were studied. Bar charts summarizing cell viability analyzed by (A) MTT assay and (B) trypan blue assay. The percentage of PLC-v and PLC-k3 cells (C) in early apoptosis and (D) distributed in each phase of the cell cycle. Results are presented as the mean \pm standard deviation of three separate experiments. ${ }^{*} \mathrm{P}<0.05$ vs. untreated PLC-v cells and ${ }^{* *} \mathrm{P}<0.05$ vs. untreated PLC-k3 at the respective time point.

was observed in the cisplatin-treated PLC-v group (36\% vs. the untreated PLC-v group) and the cisplatin-treated PLC-k3 group (38\% vs. the untreated PLC-k3 group). Following the 48-h treatment, a significant increase of $100 \%$ was observed in the PLC-v and PLC-k3 cisplatin-treated groups compared with the untreated PLC-v and PLC-k3 groups. Therefore, there was no significant difference in the levels of cisplatin-induced apoptosis between the PLC-v and PLC-k3 cells at either 24 or $48 \mathrm{~h}$.

To study the effect of survivin depletion in cisplatin treatment on cell cycle progression, flow cytometric analysis was performed. Cell cycle distributions of the PLC-v and PLC-k3 were analyzed (Fig. 2D). When cells were treated for $24 \mathrm{~h}$, no significant difference in the distribution of the cell cycle stages was observed. However, changes in the cell cycle progression of the PLC-v and PLC-k3 cells were observed when treated for $48 \mathrm{~h}$. In the PLC-v group, the distribution of cells was shifted from the G1 phase to the S phase with cisplatin treatment (a 1.6-fold increase in the percentage of cells in the $\mathrm{S}$ phase vs. the untreated cells). As for the PLC-k3 cells, a more marked shift of cells from the G1 and $\mathrm{G} 2$ phases to the $\mathrm{S}$ phase was observed with cisplatin treatment (a 2.28-fold increase in the percentage of cells in the $\mathrm{S}$ phase vs. the untreated PLC-k3 cells). Therefore, cisplatin treatment arrested cells in the $\mathrm{S}$ phase and the accumulation of cells was more profound in the survivin-depleted group.

Tumorigenicity of HCC cells with knockdown of the survivin gene in vivo. For the in vivo experiments, subcutaneous injection of the PLC-v and PLC-k3 cells was performed (Fig. 3A). The PLC-v cells successfully induced tumor growth in all mice
A
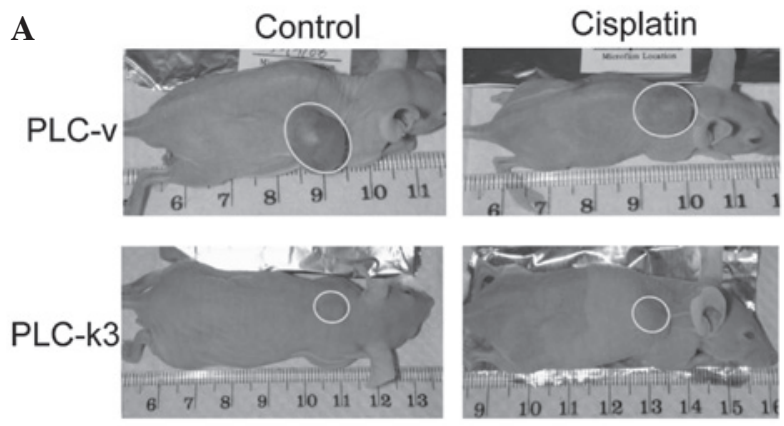

B
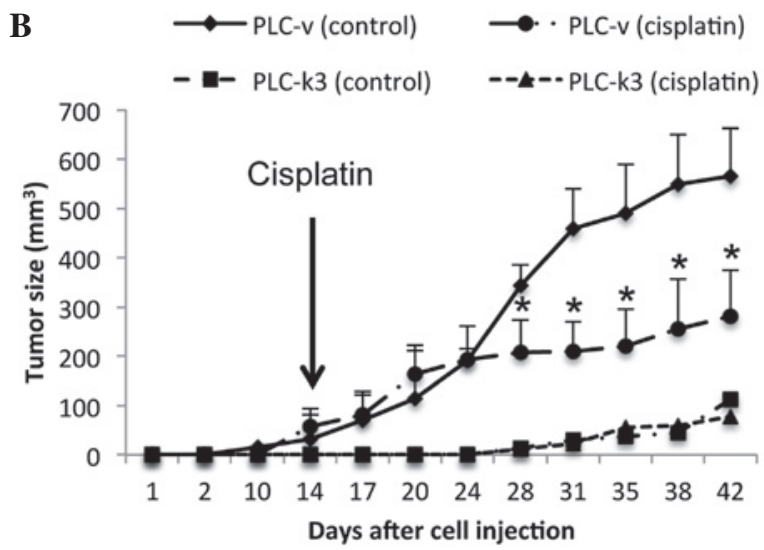

Figure 3. Survivin depletion of PLC/PRF/5 cells inhibits subcutaneous tumor formation. (A) Representative images of mice demonstrating the sizes of tumors formed with/without treatment with cisplatin, 42 weeks post-subcutaneous injection of PLC-v or PLC-k3 cells. (B) Graph summarizing tumor sizes in PLC-v- and PLC-k3-inoculated mice with/without cisplatin treatment. Results are presented as the mean \pm standard deviation of tumor sizes in six mice in the PLC-v groups and the PLC-k3 (cisplatin) group, and as the mean of tumor sizes in 2 mice in the PLC-k3 (control) group. ${ }^{*} \mathrm{P}<0.05$ vs. PLC-v (control) group. 
(12/12 mice), whereas the PLC-k3 cells only induced tumor growth in 2 of the 12 mice. In the PLC-v (control) group, the tumors grew rapidly and reached an average size of $500 \mathrm{~mm}^{3}$ prior to day 40 (Fig. 3B). In the PLC-k3 (control) group, the tumors only became visible after day 28 and grew much more slowly than those in the PLC-v (control) group. Thus, survivin depletion cannot only inhibit the growth of cells in vitro, but also prevent tumor growth in vivo.

For the cisplatin treatment groups, cisplatin was administered starting on day 14, and significantly inhibited tumor growth in the mice in the PLC-v (cisplatin) group from day 28 compared with that in the PLC-v (control) group. As the PLC-k3 cells induced tumors in only 2 mice and the tumors grew too slowly, no difference in the tumor size with cisplatin treatment was observed by 42 days post-cell injection.

\section{Discussion}

HCC is a common type of cancer worldwide and the development of an effective treatment for HCC is required. Although chemotherapeutic drugs provide $\mathrm{HCC}$ patients with prolonged survival, chemoresistance often occurs. Thus, identification of the key components of tumorigenesis and chemoresistance in HCC may provide useful information on the molecular mechanisms of hepatocarcinogenesis and chemosensitivity. These components may serve as therapeutic targets of future treatments for HCC.

Survivin, which is differentially expressed in tumors compared with that in normal adult tissues, has been suggested to be associated with poor survival rates in several types of cancer, including non-small cell lung cancer (16), colorectal cancer (10) and neuroblastoma (17). Increased survivin expression has also been associated with poor prognostic parameters and outcomes in HCC (18). Therefore, survivin may be important during tumorigenesis and in controlling chemosensitivity in HCC. In the present study, depletion of survivin in an HCC cell line (PLC-k3) was used to demonstrate its role in tumorigenesis and chemosensitivity in HCC.

The survivin-depleted cells were demonstrated to have a significantly lower viability and longer doubling time than those of the control cells. This implies that survivin depletion could inhibit cell growth and survival in vitro. The difference in cell viability between the PLC-k3 and PLC-v groups may be due to either the change in cellular apoptosis and/or changes in cell cycle progression. In the Annexin V apoptosis assay, no differences between the levels of early apoptotic cells in the PLC-k3 and PLC-v groups were observed. This implies that knockdown of survivin did not enhance the apoptotic activity of HCC cells. Therefore, this may not be the main cause of the reduced cell viability in $\mathrm{HCC}$, demonstrating that it differs from the response in other cancer cell lines, including cervical (19) and lung (20) cancers. However, the result is concordant with the findings of Ito et al (12) and Pizem et al (13) that survivin expression does not correlate with the apoptotic index of HCC tissues. These studies suggest that the oncogenic role of survivin may act through different pathways in different types of cancer.

Through cell cycle analysis in the current study, it was demonstrated that survivin depletion led to cell cycle arrest with a reduction in the $S$ phase population. Cell cycle arrest in the PLC-k3 cells is also supported by the downregulation of the expression levels of cyclins D1 and A. Cyclin D1 is a G1 protein, while cyclin A is produced in the G1 phase and is essential for the G1/S transition $(21,22)$. The downregulation of cyclins D1 and A reflects that cells were blocked at G1 phase and did not enter $\mathrm{S}$ phase. Thus, knockdown of survivin alone caused cell cycle arrest. The findings are concordant with those of a previous study which demonstrated that overexpression of survivin caused an increase in the $\mathrm{S}$ phase cell population (23). This consolidates the observation of the present study that survivin knockdown inhibited proliferation of PLC/PRF/5 cells via induction of cell cycle arrest.

Findings of the current study suggest that the knockdown of survivin is more critical in inhibiting cell proliferation than in inducing apoptosis. These results are consistent with those of previous studies stating a strong correlation between survivin expression and cell proliferation, but not apoptosis, in HCC (12-15). Previous studies have reported that survivin is essential in maintaining mitosis through stabilizing microtubules and mediates the targeting of the chromosomal passenger complex to the centromere. Thus, it is an important protein for controlling normal cell division via participation in chromosomal segregation and cytokinesis $(24,25)$. This explains the results in the present study demonstrating that knockdown of survivin hinders cell division and mitosis, and therefore leads to cell cycle arrest.

In the present study, depletion of survivin in HCC cells was also demonstrated to enhance the effects of cisplatin in vitro. A lower number of viable cells and a shorter reaction time to cisplatin treatment were observed in the PLC-k3 group, suggesting that the PLC-k3 cells are more sensitive to cisplatin treatment compared with the PLC-V cells. This finding is consistent with a previous study that demonstrated survivin overexpression protected gastric cancer cells from cisplatin treatment, while a mutant form of survivin sensitized the cells to it (26). Thus, survivin may be critical in chemosensitivity to cisplatin in HCC cells. In addition, $\mathrm{S}$ phase arrest was observed in the PLC-v and PLC-k3 cells following cisplatin treatment in the present study. This is consistent with the findings of several previous studies on other types of cancer cell that chemotherapeutic drugs, including cisplatin, caused growth arrest in the S phase (27-29). The extent of the increase in number of cells accumulated in the $\mathrm{S}$ phase being higher in the PLC-k3 group than that in the PLC-v group following cisplatin treatment further demonstrated the higher sensitivity of the PLC-k3 cells to cisplatin-induced growth arrest in the $\mathrm{S}$ phase compared with that of the PLC-v cells.

In the present study, experiments in an animal model further confirmed the findings of the in vitro studies, that survivin depletion inhibits cell growth and proliferation. It is therefore suggested that survivin is essential for carcinogenesis in HCC. However, the effect of survivin depletion on chemosensitivity could not be demonstrated in the in vivo model due to the low success rate of tumor induction in the PLC-k3 group.

In summary, knockdown of survivin in an HCC cell line reduced cell viability by inhibition of cellular proliferation via cell cycle arrest rather than induction of early apoptosis. The findings were in agreement with those of previous studies which demonstrated that survivin was highly associated with cell proliferation index in primary tumors (12-15). Cisplatin exerted an additive growth inhibitory effect on PLC/PRF/5 cells with 
survivin depletion in the present study by arresting the cells in the $\mathrm{S}$ phase of the cell cycle. Thus, knockdown of survivin may enhance the chemosensitivity of PLC/PRF/5 cells, implying that survivin overexpression may be critical in chemoresistance of HCC. These findings provide a novel therapeutic strategy for the effective treatment of patients with HCC that exhibit chemoresistance. Furthermore, patients will benefit from the reduced dosage of cisplatin necessary to produce chemotherapeutic results when survivin is downregulated.

\section{Acknowledgements}

This study was supported by the Small Project Grant (200707176035) of the University of Hong Kong and the Collaborative Research Fund (HKU5/CRF/08) of the Research Grants Council, Hong Kong, SAR, P.R. China.

\section{References}

1. Jemal A, Bray F, Center MM, Ferlay J, Ward E and Forman D: Global cancer statistics. CA Cancer J Clin 61: 69-90, 2011.

2. LaCasse EC, Baird S, Korneluk RG and MacKenzie AE: The inhibitors of apoptosis (IAPs) and their emerging role in cancer. Oncogene 17: 3247-3259, 1998.

3. Altieri DC and Marchisio PC: Survivin apoptosis: an interloper between cell death and cell proliferation in cancer. Lab Invest 79 1327-1333, 1999.

4. Reed JC: The Survivin saga goes in vivo. J Clin Invest 108: 965-969, 2001

5. Geske FJ and Gerschenson LE: The biology of apoptosis. Hum Pathol 32: 1029-1038, 2001.

6. Ambrosini G, Adida C and Altieri DC: A novel anti-apoptosis gene, survivin, expressed in cancer and lymphoma. Nat Med 3: 917-921, 1997.

7. Adida C, Crotty PL, McGrath J, Berrebi D, Diebold J and Altieri DC: Developmentally regulated expression of the novel cancer anti-apoptosis gene survivin in human and mouse differentiation. Am J Pathol 152: 43-49, 1998.

8. Tamm I, Wang Y, Sausville E, et al: IAP-family protein survivin inhibits caspase activity and apoptosis induced by Fas (CD95), Bax, caspases, and anticancer drugs. Cancer Res 58: 5315-5320, 1998.

9. Sun PL, Jin Y, Kim H, et al: Survivin expression is an independent poor prognostic marker in lung adenocarcinoma but not in squamous cell carcinoma. Virchows Arch 463: 427-436, 2013.

10. Kawasaki H, Altieri DC, Lu CD, Toyoda M, Tenjo T and Tanigawa N: Inhibition of apoptosis by survivin predicts shorter survival rates in colorectal cancer. Cancer Res 58: 5071-5074, 1998.

11. Krieg A, Werner TA, Verde PE, Stoecklein NH and Knoefel WT: Prognostic and clinicopathological significance of survivin in colorectal cancer: a meta-analysis. PLoS One 8: e65338, 2013.

12. Ito T, Shiraki K, Sugimoto K, et al: Survivin promotes cell proliferation in human hepatocellular carcinoma. Hepatology 31 1080-1085, 2000.
13. Pizem J, Marolt VF, Luzar B and Cör A: Proliferative and apoptotic activity in hepatocellular carcinoma and surrounding non-neoplastic liver tissue. Pflugers Arch 442 (6 Suppl 1): R174-R176, 2001.

14. Ikeguchi M, Hirooka Y and Kaibara N: Quantitative analysis of apoptosis-related gene expression in hepatocellular carcinoma. Cancer 95: 1938-1945, 2002.

15. Ikeguchi M, Ueta T, Yamane Y, Hirooka Y and Kaibara N: Inducible nitric oxide synthase and survivin messenger RNA expression in hepatocellular carcinoma. Clin Cancer Res 8: 3131-3136, 2002.

16. Monzó M, Rosell R, Felip E, et al: A novel anti-apoptosis gene: Re-expression of survivin messenger RNA as a prognosis marker in non-small-cell lung cancers. J Clin Oncol 17: 2100-2104, 1999.

17. Nakagawara A: Molecular basis of spontaneous regression of neuroblastoma: role of neurotrophic signals and genetic abnormalities. Hum Cell 11: 115-124, 1998.

18. Fields AC, Cotsonis G, Sexton D, Santoianni R and Cohen C: Survivin expression in hepatocellular carcinoma: correlation with proliferation, prognostic parameters, and outcome. Mod Pathol 17: 1378-1385, 2004.

19. Ambrosini G, Adida C, Sirugo G and Altieri DC: Induction of apoptosis and inhibition of cell proliferation by survivin gene targeting. J Biol Chem 273: 11177-11182, 1998.

20. Olie RA, Simões-Wüst AP, Baumann B, et al: A novel antisense oligonucleotide targeting survivin expression induces apoptosis and sensitizes lung cancer cells to chemotherapy. Cancer Res 60: 2805-2809, 2000.

21. Pagano M, Pepperkok R, Verde F, Ansorge W and Draetta G: Cyclin $\mathrm{A}$ is required at two points in the human cell cycle. EMBO J 11: 961-971, 1992.

22. Stacey DW: Cyclin D1 serves as a cell cycle regulatory switch in actively proliferating cells. Curr Opin Cell Biol 15: 158-163, 2003.

23. Suzuki A, Hayashida M, Ito T, et al: Survivin initiates cell cycle entry by the competitive interaction with Cdk4/p16(INK4a) and Cdk2/cyclin E complex activation. Oncogene 19: 3225-3234, 2000.

24. Vong QP, Cao K, Li HY, Iglesias PA and Zheng Y: Chromosome alignment and segregation regulated by ubiquitination of survivin. Science 310: 1499-1504, 2005.

25. Vader G, Kauw JJ, Medema RH and Lens SM: Survivin mediates targeting of the chromosomal passenger complex to the centromere and midbody. EMBO Rep 7: 85-92, 2006.

26. Nakamura M, Tsuji N, Asanuma K, et al: Survivin as a predictor of cis-diamminedichloroplatinum sensitivity in gastric cancer patients. Cancer Sci 95: 44-51, 2004.

27. Wang X, Wong SC, Pan J, et al: Evidence of cisplatin-induced senescent-like growth arrest in nasopharyngeal carcinoma cells. Cancer Res 58: 5019-5022, 1998.

28. Joe AK, Liu H, Suzui M, Vural ME, Xiao D and Weinstein IB: Resveratrol induces growth inhibition, S-phase arrest, apoptosis, and changes in biomarker expression in several human cancer cell lines. Clin Cancer Res 8: 893-903, 2002.

29. Lee BJ, Chon KM, Kim YS, et al: Effects of cisplatin, 5-fluorouracil, and radiation on cell cycle regulation and apoptosis in the hypopharyngeal carcinoma cell line. Chemotherapy 51: 103-110, 2005. 